\title{
Zoledronic acid inhibits the pentose phosphate pathway through attenuating the Ras-TAp73-G6PD axis in bladder cancer cells
}

\author{
XIAOLIN WANG ${ }^{1,2^{*}}$, GUANG WU ${ }^{3 *}$, GUANGXIN CAO ${ }^{2}$, LEI YANG $^{4}$, \\ HAIFEI XU ${ }^{2}$, JIAN HUANG ${ }^{2}$ and JIANQUAN HOU ${ }^{1}$ \\ ${ }^{1}$ Department of Urology, First Affiliated Hospital, Soochow University, Suzhou, Jiangsu 215006; \\ ${ }^{2}$ Department of Urology, Nantong Tumor Hospital, Nantong, Jiangsu 226361; \\ ${ }^{3}$ Department of Urology, First People's Hospital of Wujiang, Suzhou, Jiangsu 215200; \\ ${ }^{4}$ Department of Medical Oncology, Nantong Tumor Hospital, Nantong, Jiangsu 226361, P.R. China
}

Received September 15, 2014; Accepted May 29, 2015

DOI: $10.3892 / \mathrm{mmr} .2015 .3995$

\begin{abstract}
Zoledronic acid (ZA) is the current standard of care for the therapy of patients with bone metastasis or osteoporosis. ZA inhibits the prenylation of small guanosine-5'-triphosphate (GTP)-binding proteins, such as Ras, and thus inhibit Ras signaling. The present study demonstrated that ZA inhibited cell proliferation and the pentose phosphate pathway (PPP) in bladder cancer cells. In addition, the expression of glucose-6-phosphate dehydrogenase (G6PD, the rate-limiting enzyme of the PPP) was found to be inhibited by ZA. Furthermore, the stability of TAp73, which activates the expression G6PD was decreased in zoledronic acid treated cells. Decreased levels of Ras-GTP and phosphorylated-extracellular signal-regulated kinase $1 / 2$ were also observed following treatment with ZA. This may be due to the fact that activated Ras was reported to stabilize TAp73 inducing its accumulation. The inhibition of Ras activity by PT inhibitor II also significantly reduced the levels of TAp73 and G6PD and the PPP flux. Moreover, knockdown of TAp73, attenuated the PPP flux and eliminated the affection of ZA on the PPP flux. In conclusion, it was proposed that ZA can inhibit stability of TAp73 and attenuate the PPP via blocking Ras signaling in bladder cancer cells.
\end{abstract}

\section{Introduction}

Bladder cancer (BC) is a common malignancy of the urinary tract $(1,2)$. Bone metastasis occurs in $\sim 40 \%$ of patients with

Correspondence to: Professor Jianquan Hou, Department of Urology, First Affiliated Hospital, Soochow University, 188 Shizi Road, Suzhou, Jiangsu 215006, P.R. China

E-mail: jianquanhou@163.com

${ }^{*}$ Contributed equally

Key words: zoledronic acid, pentose phosphate pathway, TAp73, glucose-6-phosphate dehydrogenase, ras
BC $(3,4)$. Zoledronic acid (ZA) is effective in patients with bone metastases from bladder cancer (5-7). In addition to its bone-protective effects, ZA can prevent tumor progression (8). Due to its inhibition of the prenylation of small GTP-binding proteins, such as Ras, ZA inactivates Ras signaling and inhibits Ras-dependent cell proliferation (9-11).

P73 was identified to be a member of the p53 family and is frequently overexpressed in human tumors (12-14). Although p53 is firmly established to be a tumor suppressor, the role of p73 in human tumorigenesis is not well understood $(15,16)$. The p73 gene contains two promoters and thus encodes the transcriptional domain-containing (TAp73) and the amino deleted $(\Delta \mathrm{Np} 73)$ isoforms (17). TAp73 isoforms contain an amino-terminal transactivation domain and thus can activate the promoters of p53-target genes and induce apoptosis $(18,19) . \Delta \mathrm{Np} 73$ isoforms, which lack the transactivation domain of TAp73 protein and retain the DNA-binding and oligomerization domains, act as dominant-negative inhibitors for p53 family members by forming inactive hetero-oligomers or competing for p53-DNA-binding (20).

During rapid cell growth, adequate levels of intracellular nicotinamide adenine dinucleotide phosphate (NADPH), generated predominantly through the pentose phosphate pathway (PPP), are critical for cell survival. NADPH is required for DNA, protein and lipid biosynthesis, and is also required to generate sufficient material to support cancer cell proliferation (21-23). The tumor suppressor p53 was reported to bind to glucose-6-phosphate dehydrogenase (G6PD), the rate-limiting enzyme of the PPP, and inhibit its activity. Thus, p53 can suppress the glucose consumption and NADPH production of cells (24). Conversely, TAp73 was reported to activate the expression of G6PD and thus promote the PPP flux and NADPH production (25). The present study investigated the effects of ZA on the PPP flux and the proliferation of tumor cells.

\section{Materials and methods}

Reagents. ZA and cycloheximide ( $\mathrm{CHX}$ ) were purchased from Sigma-Aldrich (St. Louis, MO, USA). The mouse monoclonal anti-human Ras (05-1072; 1:1,000 dilution) and rabbit 
monoclonal anti-human phospho-extracellular signal-regulated kinase (ERK) 1/2 (Thr202/Tyr204 and Thr185/Tyr187; 05-797R; 1:1,000 dilution) were obtained from Millipore (Bedford, MA, USA). The rabbit polyclonal anti-human G6PD (8866; 1:1,000 dilution) and $\beta$-actin (4967; 1:3,000 dilution) antibodies were obtained from Cell Signaling Technology Inc. (Beverly, MA, USA). Rabbit polyclonal anti-human p73 (ab137797; 1/800 dilution) was from Abcam (Cambridge, MA, USA). FPT inhibitor II was purchased from Santa Cruz Biotechnology Inc. (Dallas, TX, USA). The real-time PCR Master Mix kit (SYBR green PCR master mix) was purchased from Takara Bio Inc. (Dalian, China). To evaluate Ras activity [Ras-guanosine-5'-triphosphate (GTP) levels], affinity precipitation of active Ras was performed using a Ras activation assay according to the manufacturer's instructions (Millipore).

Cell culture. T24 human bladder cancer cells and 293T cells were purchased from the Type Culture Collection of the Chinese Academy of Sciences (Shanghai, China). Cells were cultured in RPMI-1640 medium (Invitrogen Life Technologies, Carlsbad, CA, USA) supplemented with $10 \%$ fetal bovine serum, penicillin $(100 \mathrm{U} / \mathrm{ml})$ and streptomycin $(100 \mu \mathrm{g} / \mathrm{ml})$ (all from Invitrogen Life Technologies) in a humidified atmosphere of $5 \% \mathrm{CO}_{2}$ at $37^{\circ} \mathrm{C}$.

$m R N A$ extract and reverse transcription-quantitative polymerase chain reaction ( $R T-q P C R)$. To examine the expression of G6PD and TAp73 mRNA in T24 cells, RT-qPCR was performed. Total RNA was extracted from the cell lines using the RNAiso reagent (Takara, Otsu, Japan). The first-strand cDNA was generated using TransScript First-Strand cDNA Synthesis SuperMix kit (Transgen, Beijing, China). Amplification and data acquisition were run on a real-time PCR system (ABI Prism 7500; Applied Biosystems, Foster City, CA, USA) for SYBR green PCR master mix. The quantity of the cDNA sample was $1 \mu \mathrm{l}$. RT-PCR was performed by 3 min incubation at $95^{\circ} \mathrm{C}$ and 40 amplification cycles $\left(95^{\circ} \mathrm{C}\right.$ for $10 \mathrm{sec} ; 56^{\circ} \mathrm{C}$ for $15 \mathrm{sec}$; and $72^{\circ} \mathrm{C}$ for $35 \mathrm{sec}$ ). $\beta$-actin served as a control. The primer sequences used were as follows: Forward: 5'-GTACCACTGGCATCGTGATGGACT-3' and reverse 5'-CCGCTCATTGCCAATGGTGAT-3' for $\beta$-actin; forward: 5'-TGCCCCCGACCGTCTAC-3' and reverse: 5'-ATGCGG TTCCAGCCTATCTG-3' for G6PD; and forward: 5'-GCA CCTACTTTGACCTCCCC-3' and reverse: 5'-GCACTGCTG AGCAAATTGAAC-3' for TAp73.

Metabolism assays. Glucose consumption was measured in the cell lysates with Glucose Uptake Colorimetric Assay kit from (BioVision, San Francisco, CA, USA). The production of NADPH was measured in the cell lysates with NAD ${ }^{+} / \mathrm{NADH}$ Quantification Colorimetric kit (BioVision, San Francisco, CA, USA). Following the manufacturer's instructions for these measures.

Vector and cell transfection. pCMV6-TAp73 (NM_005427) was obtained from OriGene (Rockville, MD, USA). For the transfection of the T24 cells, pCMV6 empty vector (OriGene, Rockville, MD, USA) and pCMV6-TAp73 vector were transfected into cells using Lipofectamine ${ }^{\mathrm{TM}} 2000$ (Invitrogen Life Technologies). After $6 \mathrm{~h}$, the medium was refreshed and cultured for $48 \mathrm{~h}$. Then the cells were treated with ZA $(200 \mu \mathrm{M})$.

Viral production and infection. Expression plasmids for small hairpin (sh)RNAs of TAp73 were made in pLKO.1-puro plasmids (Sigma-Aldrich). The targeted sequences were: 5'-GGATTCCAGCATGGACGTCTT-3' (sh1) and 5'-CCA AGGGTTACAGAGCATTTA-3' (sh2). A negative control vector containing scrambled shRNA was also obtained from Sigma-Aldrich. The plasmids were prepared with a plasmid maxi kit and transfected in 293T cells (Invitrogen Life Technologies) with the Lipofectamine ${ }^{\mathrm{TM}} 2000$ to produce lentiviral particles. Then, the T24 cells were infected with the lentiviral particles for $24 \mathrm{~h}$. Cells were then selected with $2 \mathrm{mg} / \mathrm{ml}$ puromycin (Sigma-Aldrich) for $72 \mathrm{~h}$.

Western blot analysis. Cells were lysed in a radioimmunoprecipitation assay buffer containing and complete Protease/Phosphatase Inhibitor Cocktail (Cell Signaling Technology, Inc.). The lysate was centrifuged at $11,500 \mathrm{x} \mathrm{g}$ for $15 \mathrm{~min}$ at $4^{\circ} \mathrm{C}$ and the supernatant was collected. Protein concentration was determined using a Bradford protein assay kit (Bio-Rad, Hercules, CA, USA). The proteins were separated by $10 \%$ SDS-PAGE gel (RSBM, Taiyuan, China) and then transferred onto a polyvinylidene difluoride membrane (Millipore). The membranes were blocked with $10 \%$ milk in Tris-buffered saline with Tween-20 (TBST) for $1 \mathrm{~h}$ and then were incubated with antibodies overnight at $4^{\circ} \mathrm{C}$. After washing with TBST three times, the membranes were probed with horseradish peroxidase-conjugated secondary antibodies in TBST for $1 \mathrm{~h}$ at room temperature, then washed with PBST three times. The immunobinding signals were detected by a chemiluminescence kit (Millipore).

Cell proliferation analysis. Cell proliferation was examined by using a Cell Counting kit-8 (CCK-8; Dojindo, Kumamoto, Japan). Cells were plated and treated with ZA $(200 \mu \mathrm{M})$ in 96-well plates at 2,000 cells per well and cultured in growth medium for $20 \mathrm{~h}$. After $20 \mathrm{~h}, \mathrm{CCK}-8(10 \mu \mathrm{l})$ was added to each well containing $100 \mu \mathrm{l}$ RPMI-1640 medium. Then the plate was incubated for $2 \mathrm{~h}$ at $37^{\circ} \mathrm{C}$. Absorbance was measured at $450 \mathrm{~nm}$ using a microplate reader (SpectraMax M5 Microplate Reader; BioTek, Winooski, VT, USA).

Statistical analysis. Data were analyzed using Student's t-test. Graphpad 6.01 Prism software (GraphPad, Inc., La Jolla, CA, USA) was used for statistical analyses. $\mathrm{P}<0.05$ was considered to indicate a statistically significant difference.

\section{Results}

ZA inhibits the proliferation of bladder cancer cells and attenuates the PPP. To confirm the effects of ZA on the proliferation of bladder cancer cells, T24 human bladder cancer cell lines were treated with ZA $(200 \mu \mathrm{M})$ for $20 \mathrm{~h}$. As shown in Fig. 1A, ZA significantly reduced the proliferative activity of T24 cells. The PPP and NADPH produced in the PPP are required for rapid cancer cell growth. Thus, it was examined whether ZA affects the PPP flux in bladder cancer cell lines. It was demonstrated that glucose consumption and NADPH were inhibited 
A

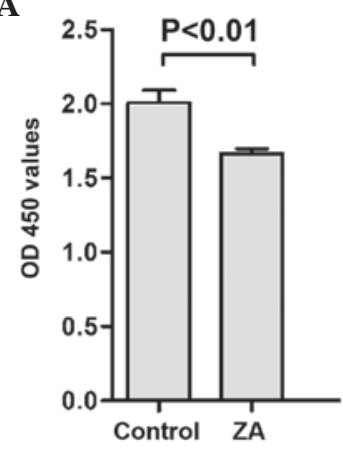

C

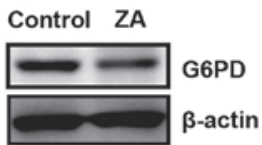

B
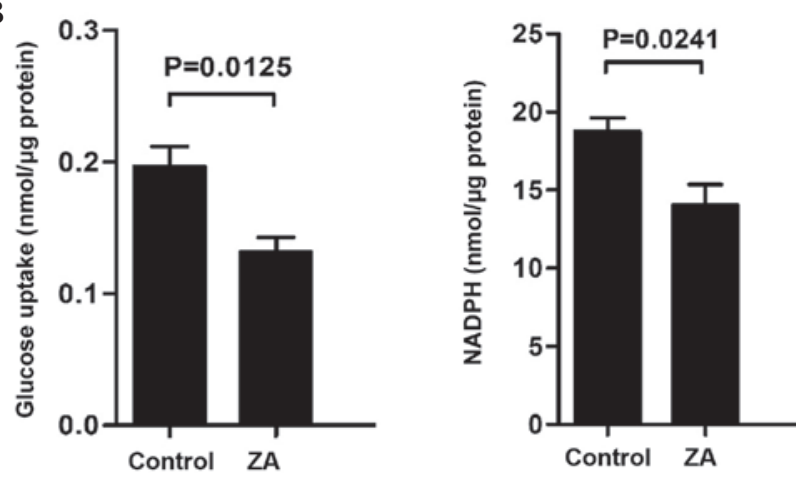

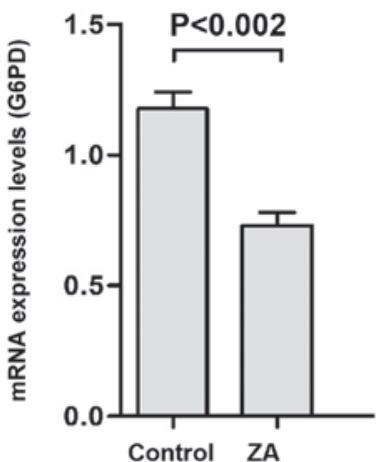

Figure 1. ZA inhibits the proliferation of bladder cancer cells and the PPP. T24 cells were treated with ZA ( $200 \mu \mathrm{M})$ for $20 \mathrm{~h}$. (A) Cell proliferation was evaluated by a cell counting kit- 8 assay. (B) Glucose uptake and NADPH levels were measured in the lysates and normalized to the total cellular protein in T24 cells treated with or without ZA. (C) The levels of G6PD were examined by western blot analysis in T24 cells treated without or with ZA (left). The levels of G6PD mRNA were examined by reverse transcription-quantitative polymerase chain reaction (right). ZA, zoledronic acid; NADPH, nicotinamide adenine dinucleotide phosphate; G6PD, glucose-6-phosphate dehydrogenase.

A

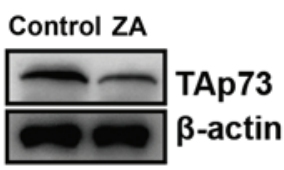

B

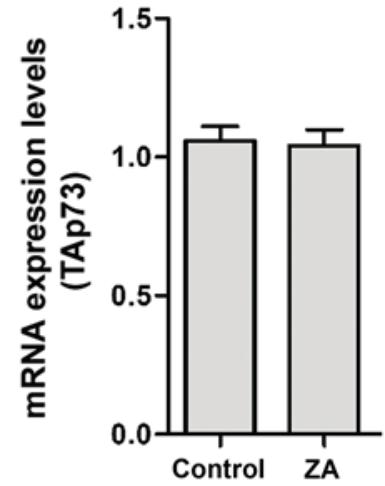

C

$\begin{array}{lllll}0 & 4 & 8 & 16 & \text { ITime (h) }\end{array}$
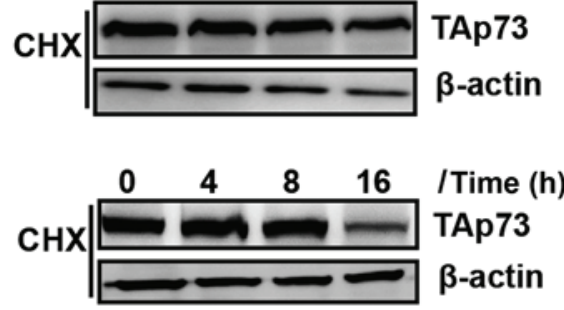

Figure 2. ZA decreases the stability of TAp73. (A) Cells were treated with ZA for $20 \mathrm{~h}$, the levels of TAp73 were examined by western blot analysis in T24 cells. (B) The levels of G6PD mRNA treated by ZA in T24 cells were examined by reverse transcription-quantitative polymerase chain reaction. (C) T24 cells were treated with or without ZA plus $50 \mu \mathrm{g} / \mathrm{ml} \mathrm{CHX}$ for the indicated times. TAp73 and $\beta$-actin were detected by western blot analysis. ZA, zoledronic acid; G6PD, glucose-6-phosphate dehydrogenase; CHX, cycloheximide.

by treatment with ZA in T24 cells (Fig. 1B). Furthermore, G6PD, the rate-limiting enzyme of the PPP, was found to be downregulated at the mRNA and protein level (Fig. 1C).

ZA decreases the stability of TAP73. TAp73 was reported to activate the expression of G6PD and thus promote the PPP flux and NADPH production (25). As shown in Fig. 2A, it was demonstrated that the levels of TAp73 decreased following treatment with ZA in T24 cells (Fig. 2A). The expression of TAp73 mRNA was then examined by RT-qPCR. The data show that there were no significant changes in TAp73 mRNA levels following treatment with ZA (Fig. 2B). It was hypothesized that the regulation of TAp73 levels by ZA may be the result of protein stability regulation. The TAp73 stability in T24 cells was then determined by treatment with $\mathrm{CHX}$. As shown in Fig. 2C, following pre-treatment with ZA, the stability of TAp73 decreased.

Stability of TAp73 regulated by ZA may depend on the inhibition of the activity of Ras. Activated Ras was reported 
A

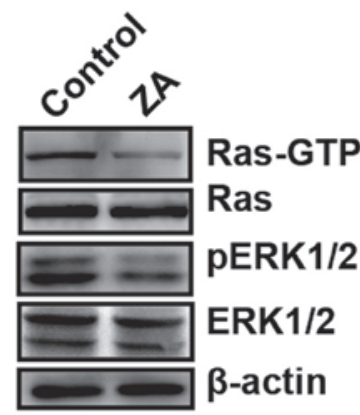

B

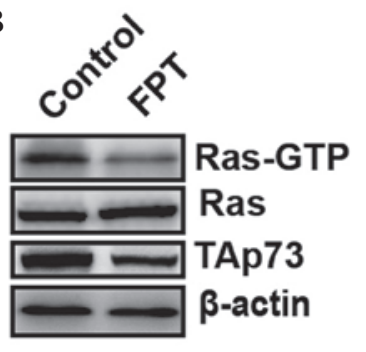

C

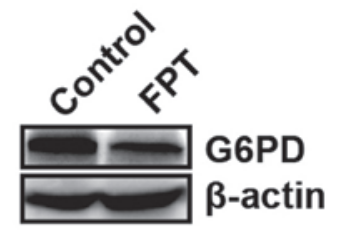

D

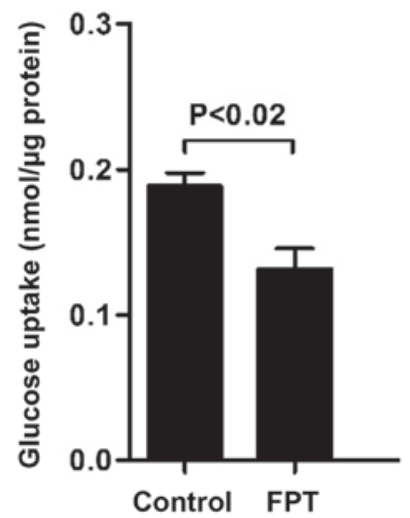

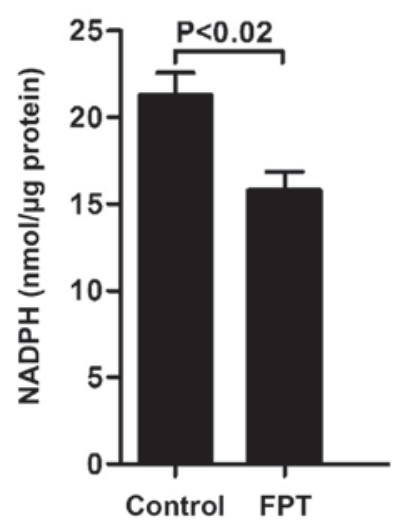

Figure 3. Stability of TAp73 regulated by ZA depends on the inhibition of Ras. (A) ZA decreased the activity of Ras, determined as the quantity of Ras-GTP and phospho-ERK 1/2 (Thr202/Tyr204, Thr185/Tyr187) in T24 cells after $20 \mathrm{~h}$ treatment. The levels of $\beta$-actin were shown to confirm equivalent protein loading. (B) Detection of Ras-GTP, Ras and TAp73 in T24 cells after 16-h incubation with or without $200 \mu \mathrm{M}$ FPT inhibitor II for 16 h. (C) After the treatment with $200 \mu \mathrm{M}$ FPT inhibitor II for $16 \mathrm{~h}$, the levels of G6PD and $\beta$-actin were examined by western blot analysis. (D) Glucose uptake and NADPH levels were measured in lysates and normalized to total cellular protein amount in T24 cells treated without or with $200 \mu \mathrm{M}$ FPT inhibitor II for $16 \mathrm{~h}$. ERk, extracellular-signal regulated kinase; NADPH, nicotinamide adenine dinucleotide phosphate.
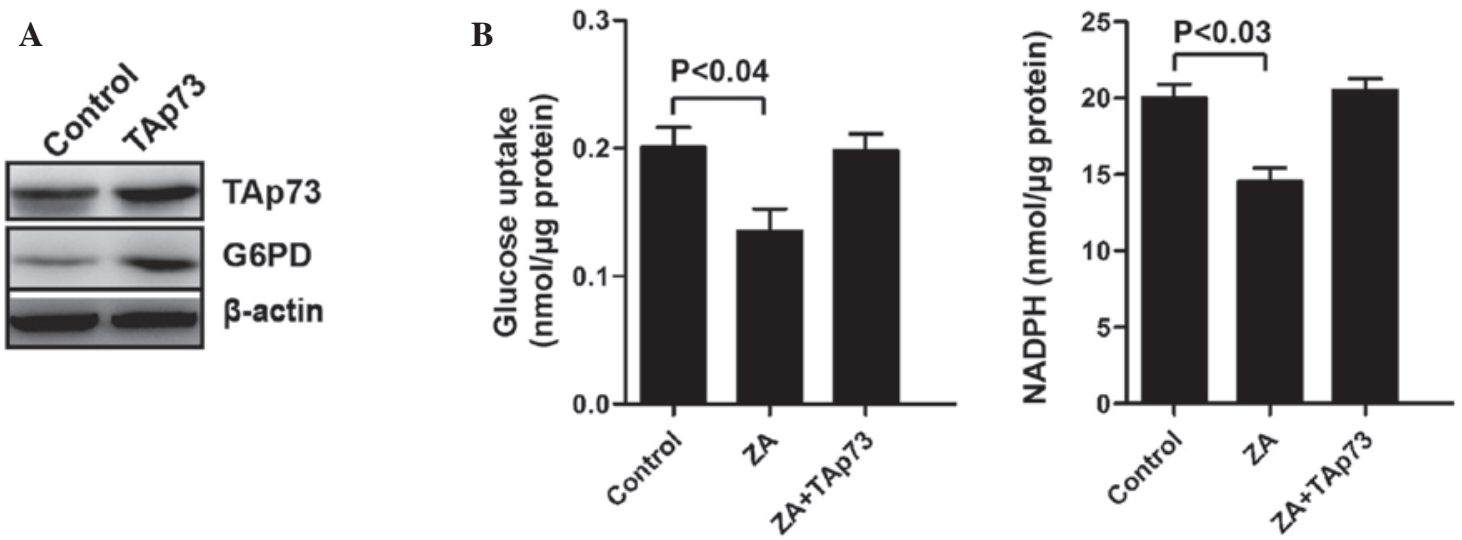

Figure 4. Inhibitory effect of the pentose phosphate pathway by ZA is attenuated by TAp73 overexpression. (A) T24 cells were transfected with empty control and TAp73 vectors for $48 \mathrm{~h}$. The expression of TAp73, G6PD and $\beta$-actin were analyzed by western blot analysis. (B) After transfection with empty control (control and ZA) or TAp73 (ZA+TAp73) vectors for 48 h, T24 cells were treated with or without ZA (200 $\mu$ M) for 20 h. Glucose uptake and NADPH levels were measured in lysates and normalized to total cellular protein amount. ZA, zoledronic acid; NADPH, nicotinamide adenine dinucleotide phosphate; G6PD, glucose-6-phosphate dehydrogenase.

to stabilize TAp73 inducing its accumulation (26). The activity of Ras in T24 cells treated with ZA was then examined. GTP-bound Ras (Ras-GTP), a marker of Ras activation, as well as the quantity of ERK1/2 phosphorylation were decreased in T24 cells following treatment with ZA for 20 h (Fig. 3A). FPT inhibitor II, exhibited inhibitory effects of Ras farnesylation and activity, was substituted for
ZA to treat T24 cells. As shown in Fig. 3B, FPT inhibitor II significantly reduced the levels of Ras-GTP and TAp73. In addition, FPT inhibitor II also inhibited the expression of G6PD (Fig. 3C), glucose consumption and NADPH production (Fig. 3D). These results support the role of ZA in the regulation of the stability of TAp73 via inhibiting the activity of Ras. 
A

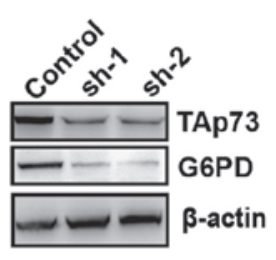

C

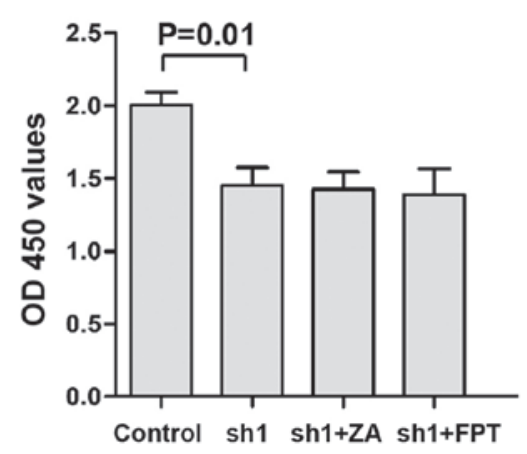

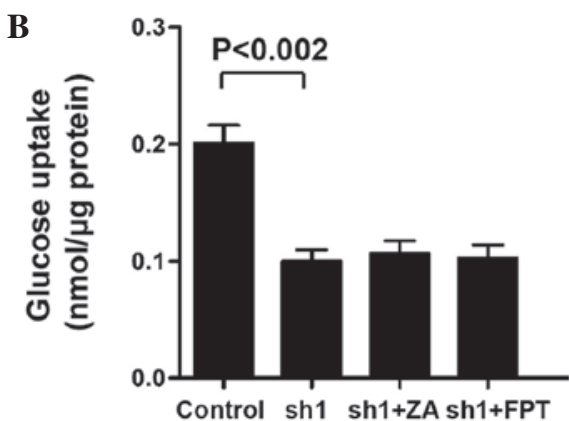

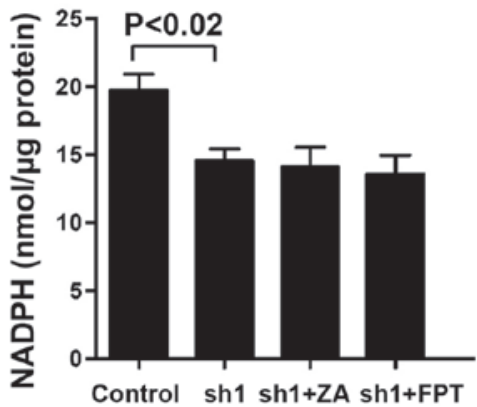

Figure 5. Knockdown of TAp73 inhibits the pentose phosphate pathway of T24 cells that is independent of Ras and ZA. (A) T24 cells were stably transfected with scramble control and two different shRNAs targeting TAp73 (sh1 and sh2). The expression of TAp73, G6PD and $\beta$-actin were analyzed by western blot analysis. (B) Stable TAp73-knockdown T24 cells with sh1 were treated with ZA or FPT inhibitor II. Glucose uptake and NADPH levels were measured in lysates and normalized to total cellular protein. (C) Stable TAp73-knockdown T24 cells with sh1 were treated with ZA or FPT inhibitor II. The cell proliferation were evaluated by cell counting kit-8. ZA, zoledronic acid; shRNA, small hairpin RNA; G6PD, glucose-6-phosphate dehydrogenase.

$Z A$ regulates $P P P$ through the regulation of TAp73. In order to further investigate the function of $\mathrm{ZA}$ in the regulation of the PPP. Following transfection with TAp73 for $48 \mathrm{~h}$ (Fig. 4A), the inhibitory effect on the PPP by ZA, as well as the glucose consumption and NADPH production was attenuated by TAp73 overexpression (Fig. 4B). Furthermore, the expression of G6PD was also upregulated (Fig. 4A). These results further support that ZA inhibits the expression of G6PD and then attenuates the PPP through the downregulation of TAp73.

Knockdown of TAp73 inhibits the PPP of T24 cells independent of Ras activity or ZA treatment. shRNA-mediated TAp73 knockdown was then performed in T24 cells and the PPP flux was examined. It was demonstrated that the expression of G6PD (Fig. 5A) and the PPP flux (Fig. 5B) were inhibited following knockdown of TAp73 in T24 cells. Moreover, FPT inhibitor II and ZA did not affect the PPP flux after the knockdown of TAp73 (Fig. 5B). Similarly, the proliferation of T24 cells was not significantly changed by the FPT inhibitor II or ZA after the knockdown of TAp73 (Fig. 5C). These results confirm the role of TAp73 in the inhibition of the PPP flux by ZA.

\section{Discussion}

In the present study, it was demonstrated that ZA inhibited the proliferation of bladder cancer cells and the PPP. Moreover, ZA was found to decrease the stability of TAp73, which activates the expression of G6PD (the rate-limiting enzyme of PPP). Decreased levels of Ras-GTP and p-ERK1/2 were also found to be associated with the treatment with ZA. Furthermore, the inhibition of Ras activity by PT Inhibitor II significantly reduced the levels of TAp73, G6PD, glucose consumption and NADPH production.

$\mathrm{ZA}$ is effective in patients with bone metastases from bladder cancer. In addition to its bone-protective effects, ZA can prevent tumor progression (27). ZA was reported to inhibit the prenylation of small GTP-binding proteins, such as Ras. Thus, ZA was hypothesized to inactivate Ras signaling and inhibit Ras-dependent cell proliferation $(9,28)$. Previous studies have shown that ZA inhibited the activity of Ras and hence inhibit the expression of hypoxia-inducible factor 1- $\alpha$ (HIF1A) (29). NADPH is required for DNA, protein and lipid biosynthesis during the rapid cell growth of cancer cells. The PPP is the predominant source of NADPH and it is often enhanced during cell cycle progression of cancer cells $(23,30)$.

In this study, the PPP flux was found to be inhibited by ZA. In addition, the expression of G6PD, the rate-limiting enzyme of the PPP, was also found to be inhibited by ZA. Activated Ras was reported to stabilize TAp73 protein, ZA was shown to decrease the levels of Ras-GTP and p-ERK1/2 with treatment with ZA. FPT inhibitor II is the inhibitor of Ras farnesylation and its activity. Similar to ZA, FPT inhibitor II significantly reduced the levels of Ras-GTP, G6PD, TAp73 and the PPP flux. It was also demonstrated that knockdown of TAp73 resulted in an inhibition of the PPP flux. Moreover, ZA could not regulate the PPP flux in TAp73 knockdown cells. These results implied that ZA inhibits the PPP flux and the expression of G6PD via blocking Ras signaling and attenuating the stability of TAp73 in bladder cancer cells. The findings of the present study revealed a novel mechanism for ZA to regulate the PPP. Besides the bone-protective effects, ZA restrained the PPP flux of cancer 
cells and inhibited their proliferation. The mechanistic target of ZA was TAp73, and thus, TAp73-overexpressing tumors may show enhanced sensitivity to ZA. Due to its targeting of TAp73, ZA may potentially be utilized in cancer therapies.

\section{References}

1. Parkin DM: The global burden of urinary bladder cancer. Scand J Urol Nephrol (Suppl): 12-20, 2008.

2. Noon AP, Albertsen PC, Thomas F, Rosario DJ and Catto JW: Competing mortality in patients diagnosed with bladder cancer: Evidence of undertreatment in the elderly and female patients. $\mathrm{Br}$ J Cancer 108: 1534-1540, 2013.

3. Zarogoulidis K, Boutsikou E, Zarogoulidis P, Eleftheriadou E, Kontakiotis T, Lithoxopoulou H, Tzanakakis G, Kanakis I and Karamanos NK: The impact of zoledronic acid therapy in survival of lung cancer patients with bone metastasis. Int J Cancer 125: 1705-1709, 2009.

4. Hirata H, Hinoda Y, Ueno K, Shahryari V, Tabatabai ZL and Dahiya R: MicroRNA-1826 targets VEGFC, beta-catenin (CTNNB1) and MEK1 (MAP2K1) in human bladder cancer. Carcinogenesis 33: 41-48, 2012.

5. Alcaraz A, González-López R, Morote J, de la Piedra C, Meseguer C, Esteban E, Climent M, González-Gragera B, Alvarez-Ossorio JL, Chirivella I, et al: Biochemical markers of bone turnover and clinical outcome in patients with renal cell and bladder carcinoma with bone metastases following treatment with zoledronic acid: The TUGAMO study. Br J Cancer 109: 121-130, 2013

6. Zaghloul MS, Boutrus R, El-Hossieny H, Kader YA, El-Attar I and Nazmy M: A prospective, randomized, placebo-controlled trial of zoledronic acid in bony metastatic bladder cancer. Int J Clin Oncol 15: 382-389, 2010.

7. Saad F and Eastham JA: Zoledronic acid use in patients with bone metastases from renal cell carcinoma or bladder cancer. Semin Oncol (37 Suppl 1): S38-S44, 2010.

8. Gouin F, Ory B, Rédini F and Heymann D: Zoledronic acid slows down rat primary chondrosarcoma development, recurrent tumor progression after intralesional curretage and increases overall survival. Int J Cancer 119: 980-984, 2006.

9. Goffinet M, Thoulouzan M, Pradines A, Lajoie-Mazenc I, Weinbaum C, Faye JC and Séronie-Vivien S: Zoledronic acid treatment impairs protein geranyl-geranylation for biological effects in prostatic cells. BMC Cancer 6: 60, 2006.

10. Koto K, Murata H, Kimura S, Sawai Y, Horie N, Matsui T, Ryu K, Ashihara E, Maekawa T, Kubo T, et al: Zoledronic acid significantly enhances radiation-induced apoptosis against human fibrosarcoma cells by inhibiting radioadaptive signaling. Int J Oncol 42: 525-534, 2013.

11. Rowinsky EK, Windle JJ and Von Hoff DD: Ras protein farnesyltransferase: A strategic target for anticancer therapeutic development. J Clin Oncol 17: 3631-3652, 1999.

12. Wei J,Zaika E and Zaika A: p53 Family: Role of protein isoforms in human cancer. J Nucleic Acids 2012: 687359, 2012.

13. Stiewe $\mathrm{T}$ and Putzer BM: Role of $\mathrm{p} 73$ in malignancy: Tumor suppressor or oncogene? Cell Death Differ 9: 237-245, 2002.
14. Lunghi P, Costanzo A, Mazzera L, Rizzoli V, Levrero M and Bonati A: The p53 family protein $\mathrm{p} 73$ provides new insights into cancer chemosensitivity and targeting. Clin Cancer Res 15: 6495-6502, 2009.

15. Tomkova K, Belkhiri A, El-Rifai W and Zaika AI: p73 isoforms can induce T-cell factor-dependent transcription in gastrointestinal cells. Cancer Res 64: 6390-6393, 2004.

16. Zaika AI and El-Rifai W: The role of p53 protein family in gastrointestinal malignancies. Cell Death Differ 13: 935-940, 2006.

17. Conforti F, Yang AL, Agostini M, Rufini A, Tucci P, Nicklison-Chirou MV, Grespi F, Velletri T, Knight RA, Melino G, et al: Relative expression of TAp73 and $\triangle \mathrm{Np} 73$ isoforms. Aging (Albany NY) 4: 202-205, 2012.

18. Rufini A, Agostini M, Grespi F, Tomasini R, Sayan BS, Niklison-Chirou MV, Conforti F, Velletri T, Mastino A, Mak TW, et al: 773 in Cancer. Genes Cancer 2: 491-502, 2011.

19. Lin D, Cui Z, Kong L, Cheng F, Xu J and Lan F: p73 participates in WWOX-mediated apoptosis in leukemia cells. Int J Mol Med 31: 849-854, 2013.

20. Grob TJ, Novak U, Maisse C, Barcaroli D, Lüthi AU, Pirnia F, Hügli B, Graber HU, De Laurenzi V, Fey MF, et al: Human delta Np73 regulates a dominant negative feedback loop for TAp73 and p53. Cell Death Differ 8: 1213-1223, 2001.

21. Samudio I, Fiegl M and Andreeff M: Mitochondrial uncoupling and the Warburg effect: Molecular basis for the reprogramming of cancer cell metabolism. Cancer Res 69: 2163-2166, 2009.

22. Cairns RA, Harris IS and Mak TW: Regulation of cancer cell metabolism. Nat Rev Cancer 11: 85-95, 2011.

23. Jones RG and Thompson CB: Tumor suppressors and cell metabolism: A recipe for cancer growth. Genes Dev 23: 537-548, 2009.

24. Jiang $\mathrm{P}, \mathrm{Du} \mathrm{W}$, Wang $\mathrm{X}$, Mancuso $\mathrm{A}, \mathrm{Gao} \mathrm{X}$, Wu M and Yang $\mathrm{X}$ : p53 regulates biosynthesis through direct inactivation of glucose-6-phosphate dehydrogenase. Nat Cell Biol 13: 310-316, 2011.

25. Du W, Jiang P, Mancuso A, Stonestrom A, Brewer MD, Minn AJ, Mak TW, Wu M and Yang X: TAp73 enhances the pentose phosphate pathway and supports cell proliferation. Nat Cell Biol 15: 991-1000, 2013

26. Fernandez-Garcia B, Vaqué JP, Herreros-Villanueva M, Marques-Garcia F, Castrillo F, Fernandez-Medarde A, León J and Marín MC: p73 cooperates with Ras in the activation of MAP kinase signaling cascade. Cell Death Differ 14: 254-265, 2007.

27. Chang J, Wang W, Zhang H, Hu Y and Yin Z: Bisphosphonates regulate cell proliferation, apoptosis and pro-osteoclastic expression in MG-63 human osteosarcoma cells. Oncol Lett 4: 299-304, 2012.

28. Ohtsuka Y, Manabe A, Kawasaki H, Hasegawa D, Zaike Y, Watanabe S, Tanizawa T, Nakahata T and Tsuji K: RAS-blocking bisphosphonate zoledronic acid inhibits the abnormal proliferation and differentiation of juvenile myelomonocytic leukemia cells in vitro. Blood 106: 3134-3141, 2005.

29. Riganti C, Castella B, Kopecka J, Campia I, Coscia M, Pescarmona G, Bosia A, Ghigo D and Massaia M: Zoledronic acid restores doxorubicin chemosensitivity and immunogenic cell death in multidrug-resistant human cancer cells. PLoS One 8: e60975, 2013.

30. Cairns RA, Harris I, McCracken S and Mak TW: Cancer cell metabolism. Cold Spring Harb Symp Quant Biol 76: 299-311, 2011. 